\title{
Population pharmacokinetic analysis of meloxicam in rheumatoid arthritis patients
}

\section{Ingolf Meineke \& Dietrich Türck'}

Department of Clinical Pharmacology, Georg August Universität Göttingen, D-37075 Göttingen, and ${ }^{1}$ Department of PharmacokineticsandDrug Metabolism, Boehringer Ingelheim Pharma KG, D-88397 Biberach an der Riss, Germany

\begin{abstract}
Aim To perform a nonlinear mixed effect modelling (NONMEM) population pharmacokinetic analysis of meloxicam plasma concentrations in rheumatoid arthritis (RA) patients participating in three clinical trials, and to evaluate the effects of age, weight, gender and concomitant medications on meloxicam pharmacokinetics.

Methods Meloxicam was administered to RA patients once daily for 3 weeks or 6 months at doses between 7.5 and $60 \mathrm{mg}$. Plasma samples were obtained at least 7 days after the first dose and meloxicam plasma concentrations were quantified by h.p.l.c..
\end{abstract}

Results NONMEM analysis was conducted on plasma samples derived from 586 patients. A one-compartmental model was found to describe the data adequately. For a typical subject in the population, a clearance of $0.3771 \mathrm{~h}^{-1}(95 \%$ confidence interval (CI) $0.0304-0.449)$ in males and $0.3471 \mathrm{~h}^{-1}$ (95\% CI 0.274-0.419) in females was obtained. The volume of distribution was estimated to be 14.91 . The findings were corroborated by subsequent analysis using WinBUGS. Analysis of covariates showed that age and gender both significantly $(P<0.005)$ affected clearance. The effect of age was relatively small and a dose adjustment of $<10 \%$ was deemed unnecessary. Differences between males and females were attributed to differences in weight. No clinically relevant drug-drug interactions were found, although sulphasalazine and glucocorticoids both significantly $(P<0.005)$ affected meloxicam clearance $(+19 \%$ and $-12 \%$, respectively). The mechanisms by which these agents affect meloxicam clearance remain to be elucidated.

Conclusions The population pharmacokinetic meloxicam data from patients with RA gave similar results to those obtained from phase I trials. However, uncommon drug interactions may not be detected in phase I trials because of the small number of observations made.

Keywords: interaction, meloxicam, nonsteroidal anti-inflammatory drug

\section{Introduction}

Meloxicam is a nonsteroidal anti-inflammatory drug (NSAID) with selectivity towards COX-2 compared with COX-1 [1]. It is almost completely absorbed following oral administration with an absolute bioavailability of $89 \%$. Peak plasma concentrations are reached 5-6 h after oral dosing when taken concomitantly with a light meal, as generally recommended [2]. Meloxicam undergoes extensive metabolism, primarily by cytochrome $\mathrm{P} 450$ CYP2C9 and to a minor extent by CYP3A4 [3], form-

Correspondence: Dr Ingolf Meineke, Department of Clinical Pharmacology, University of Göttingen, Robert-Koch-Str. 40, D-37075 Göttingen, Germany. Tel.: + 4955139 5796; Fax:+49 55। 39 9652;

E-mail: imeineke@med.uni-goettingen.de

Received 18 July, accepted 26 September 2002. ing four major inactive metabolites [1, 4]. The pharmacokinetics of meloxicam are linear over the dose range $7.5-30 \mathrm{mg}$ and remain unchanged from single to multiple dosing [5]. Total meloxicam clearance is $7-8 \mathrm{ml}$ $\mathrm{min}^{-1}$ and the terminal elimination half-life is approximately $20 \mathrm{~h}$, making meloxicam suitable for once daily dosing [5]. Steady-state drug plasma concentrations are reached within 3-5 days [5]. Other NSAIDs that are administered once daily, such as piroxicam and tenoxicam, have a much longer half-life, approximately 50 and 70 h, respectively [6-8].

Meloxicam provides effective therapy for osteoarthritis $[9,10]$ and rheumatoid arthritis (RA) [11], and its gastrointestinal tolerability profile compares favourably to classical NSAIDs [12]. Many patients with rheumatic disease are elderly and have concurrent diseases that also 
require treatment. It is important therefore to identify any potential interactions between meloxicam and comedications. Therefore, a screen of meloxicam plasma concentrations in samples collected for compliance monitoring may help to identify the impact of a variety of other drugs on meloxicam pharmacokinetics (eg sulphasalazine) in addition to that of the standard covariates: gender, age and weight. This paper describes a modelling analysis of meloxicam concentrations in compliance samples (with available dosing history) from RA patients participating in three clinical trials $[13,14]$. The aim of this work was the development of a quantitative pharmacokinetic model that allows the prediction of individual clearance values based on the gender, age and weight of a patient.

\section{Methods}

A nonlinear mixed effect modelling (NONMEM) population pharmacokinetic analysis of meloxicam plasma concentrations was carried out on samples derived from patients with RA participating in three international multicentre, randomized, double-blind, group comparison trials, two of 3 weeks' duration and one of 6 months' duration.

\section{Patient selection}

Males and females aged between 18 and 80 years with RA, as defined by American Rheumatism Association (ARA) criteria, were enrolled in the two 3-week trials [15]; patients with RA as defined by the ARA revised criteria [16] were enrolled in the 6-month trial if they required anti-inflammatory treatment. The following exclusion criteria were used: overt cardiac, hepatic or renal insufficiency; untreated hypertension, allergic bronchial asthma or an acute gastrointestinal ulcer; pregnant or nursing women; known hypersensitivity to analgesics, antipyretics and NSAIDs; administration of corticosteroids during the last 6 weeks; any clinically relevant abnormal laboratory values; any other concomitant disease or therapy likely to interfere with assessment of tolerability or efficacy. The trials were conducted in accordance with the Declaration of Helsinki, the guidelines of the WHO and the German Drug Act (AMG). The protocols were reviewed and approved by the responsible ethics committees. All patients gave informed written consent prior to their inclusion.

\section{Treatment}

Meloxicam capsules (manufactured by Boehringer Ingelheim Pharma KG, Biberach, Germany) were administered once daily for 3 weeks (in two of the trials) or
6 months (in the third trial) at doses ranging from $7.5 \mathrm{mg}$ to $60 \mathrm{mg}$. Patients receiving second-line therapy had been stabilized for at least 3 months prior to inclusion in the trials. No additional antirheumatic or analgesic drug was allowed during the study (except paracetamol up to a maximum of $4.0 \mathrm{~g} \mathrm{day}^{-1}$ ). Patients underwent a washout period of 3-7 days, after which they were randomly assigned their study medication.

\section{Analysis}

Meloxicam plasma concentrations were quantified by a specific and validated high-performance liquid chromatography (h.p.l.c.) assay [4] in steady-state samples taken during days 7 and 21 in two of the trials and after treatment for 14 days, and 1,2, 3 and 6 months in the remaining trial. In brief, $50 \mu \mathrm{l}$ of a plasma sample were applied to a Bondapak C18 Corasil-filled precolumn $(20 \times 4.5 \mathrm{~mm})$ being flushed with $0.01 \mathrm{M}$ sulphuric acid. After 4 min the extracted compounds were back flushed with methanol, water, acetonitrile and glacial acetic acid $(600 / 500 / 50 / 20, \mathrm{v} / \mathrm{v} / \mathrm{v} / \mathrm{v})$ containing $1.01 \mathrm{~g} \mathrm{l}^{-1}$ heptanesulphonic acid. The separation was achieved on an ODS Hypersil column $(125 \times 4.6 \mathrm{~mm}, 5 \mu \mathrm{m})$. Eluting substances were monitored by UV detection at $355 \mathrm{~nm}$. The assay was linear in the range $0.05-7.5 \mu \mathrm{g} \mathrm{ml}^{-1}$. The limit of quantification was set at $0.05 \mu \mathrm{g} \mathrm{ml}^{-1}$. The precision within this range was typically about $10 \%$.

\section{Population pharmacokinetic analysis}

The primary aims of the analysis were to determine the clearance, volume of distribution and absorption rate constant for meloxicam in patients with RA, and to investigate the effect of age, weight and gender on meloxicam pharmacokinetics. Additionally, the effect of sulphasalazine and other comedications on meloxicam clearance was analysed. Raw data for evaluation, including patient information and drug concentration-time values, were used to create a data file. Further data items were added representing the event description of each record, steady-state concentrations and dose. One hundred and fifty observations were made between $0.1 \mathrm{~h}$ and $3 \mathrm{~h}$, and a further 290 observations between $3 \mathrm{~h}$ and $12 \mathrm{~h}$, whereas the remaining samples were taken at later times. Meloxicam has a terminal elimination half-life of 15-20 h. Therefore, steady state is achieved after 3-5 days continued treatment, and the first samples were collected after 7 days. NONMEM (version V) [17] was used for the development of the population pharmacokinetic model. The first-order conditional estimates method was employed with an exponential error model for the interindividual variability and a proportional error model for the residual variability. These statistical models reflect the 
right skewed distribution typically seen with pharmacokinetic parameters and the heteroscedasticity of h.p.l.c. assays, respectively. The final structural model, namely the pharmacokinetic model plus covariates, was then reanalysed using WinBUGS (Bayesian inference Using Gibbs Sampling) software (MRC Biostatistics Unit, Cambridge, UK) [18]. As model implementation and optimization algorithms are fundamentally different in NONMEM and WinBUGS, comparison of the respective results was used to assess the validity of the suggested model. In addition, WinBUGS is very convenient for this purpose, as the program can read and interpret NONMEM style data files.

\section{Results}

Patients

Plasma samples accompanied with sufficient documentation on drug intake and blood sampling times were available from 602 of the patients over the three trials. Of these, 586 patients (1226 samples) were included in the analysis. Sixteen patients were excluded because they showed concentration values that suggested failure to comply. Demographic data are shown in Table 1. The mean age of the patients studied was 54.5 years, and $76 \%$ were female.

\section{Pharmacokinetics}

A one-compartment model was found to describe adequately the plasma concentration data. The use of a twocompartment or a recirculation model did not result in a better fit to the data based on a comparison of the objective function values (Akaike criterion) [19]. The one-compartment model was used to assess whether the covariates had any influence on meloxicam pharmacokinetics (Table 3). Those that significantly improved the NONMEM objective function were used to build the final model using the forward-inclusion-backwardelimination approach [20].

The identity plot shows the fit of the model to the data (Figure 1), which is satisfactory for both programs. Moreover, there was good agreement between both sets of predictions, and with few exceptions the NONMEM

Table 1 Demographics of patients included in the NONMEM analysis.

\begin{tabular}{lccc}
\hline & All patients & Male & Female \\
\hline Number of patients & 586 & 141 & 445 \\
Age (years); mean (s.d.) & $54.5(12.2)$ & $55.7(11.0)$ & $54.2(12.5)$ \\
Range (years) & $18-80$ & $21-76$ & $18-80$ \\
Height (cm); mean (s.d.) & $164.8(8.6)$ & $173.3(7.9)$ & $162.0(6.9)$ \\
Weight (kg); mean (s.d.) & $68.7(12.5)$ & $76.8(11.6)$ & $66.1(11.6)$ \\
\hline
\end{tabular}

individual prediction falls within the WinBUGS credible interval.

Age and gender both significantly $(P<0.005)$ influenced clearance, so that advanced age and female gender were associated with a decrease in clearance (Figure 2), and increasing body weight with an increase in meloxicam clearance.

The final model was specified as:

$C L=\Theta_{1} \cdot(A G E / 50)^{\Theta 6}+\Theta_{4} \cdot W G T+\Theta_{7} \cdot(1-\mathrm{SEX})$

$V=\Theta_{2} . W G T$

$K A=C L / V+\Theta_{3}$

$F 1=\Theta_{5} \cdot(1-\mathrm{OCC})+\mathrm{OCC}$

where $\Theta_{i}=$ the regression parameter to be estimated; $C L$ = clearance in $1 \mathrm{~h}^{-1} ; V=$ volume of distribution in $1 ; K A$ $=$ absorption constant in $\mathrm{h}^{-1} ; F 1=$ the relative bioavailability at the first observation (occurrence $(\mathrm{OCC})=0$ ) with respect to other observations $(\mathrm{OCC}=1)$, as the absolute bioavailability cannot be estimated after oral drug administration; AGE in years; WGT = weight in $\mathrm{kg}$; SEX-male $=0$ and female $=1$.

The regression parameters for the mean subject in the population under investigation (54.5 years and $68.7 \mathrm{~kg}$ ) are shown in Table 3 , and the population pharmacokinetic information in Table 2.

Interindividual variability in clearance, as calculated from the NONMEM estimate of interindividual variance, ranged from $46 \%$ to $214 \%$ of the population mean $\left(100 \pm 1.966^{\star} \operatorname{VarCoeff}\right)$ with individual estimates ranging from 0.085 to $0.7771 \mathrm{~h}^{-1}$. Similarly, the absorption constant ranged from $4 \%$ to $3000 \%$ of the mean, with individual values between 0.06 and $01.37 \mathrm{~h}^{-1}$. In the final

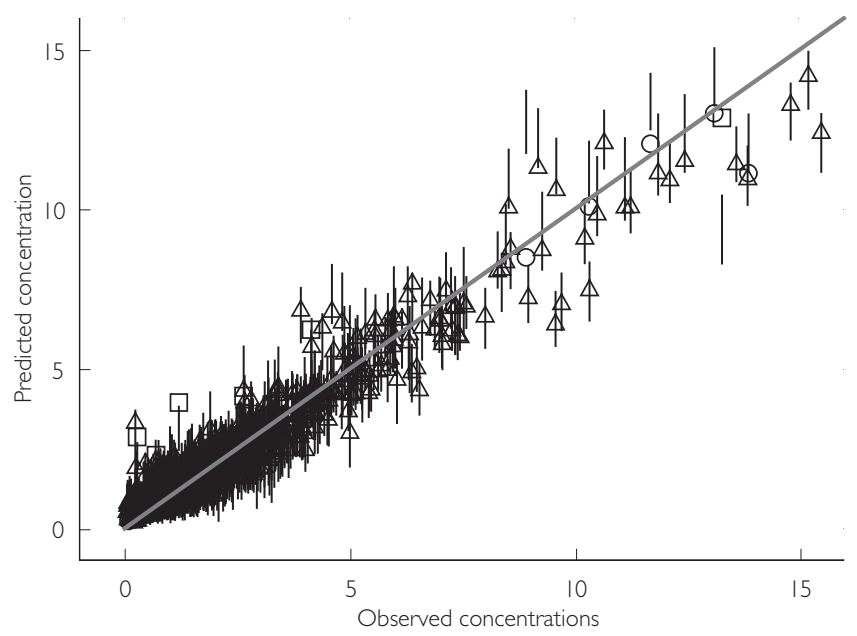

Figure 1 Identity plot: predicted vs. observed concentrations. Individual concentration predictions for both, the NONMEM fitted data and the WINBUGS fitted data are shown. $\triangle$, NONMEM predictions from the final model within the WINBUGS credible interval (-); $\bigcirc$, those below the interval; $\square$, those above the interval, respectively. 
model, only two random variables were supported by the data. Therefore, no estimates are available for the interindividual variability in volume of distribution. The individual estimates for this parameter ranged from 7.81 to 25.21 due to differences in body weights.

Using WinBUGS, the model was defined as

$\log \mathrm{CL}=\mu[1]+\mu[2] \star \mathrm{SEX}+\mu[3] \star \mathrm{AGE}+\mu[4]^{\star} \mathrm{WGT}$

$\log \mathrm{V}=\mu[5]+\mu[6] \star \mathrm{WGT}$

$\log K A^{\star}=\mu[7]$

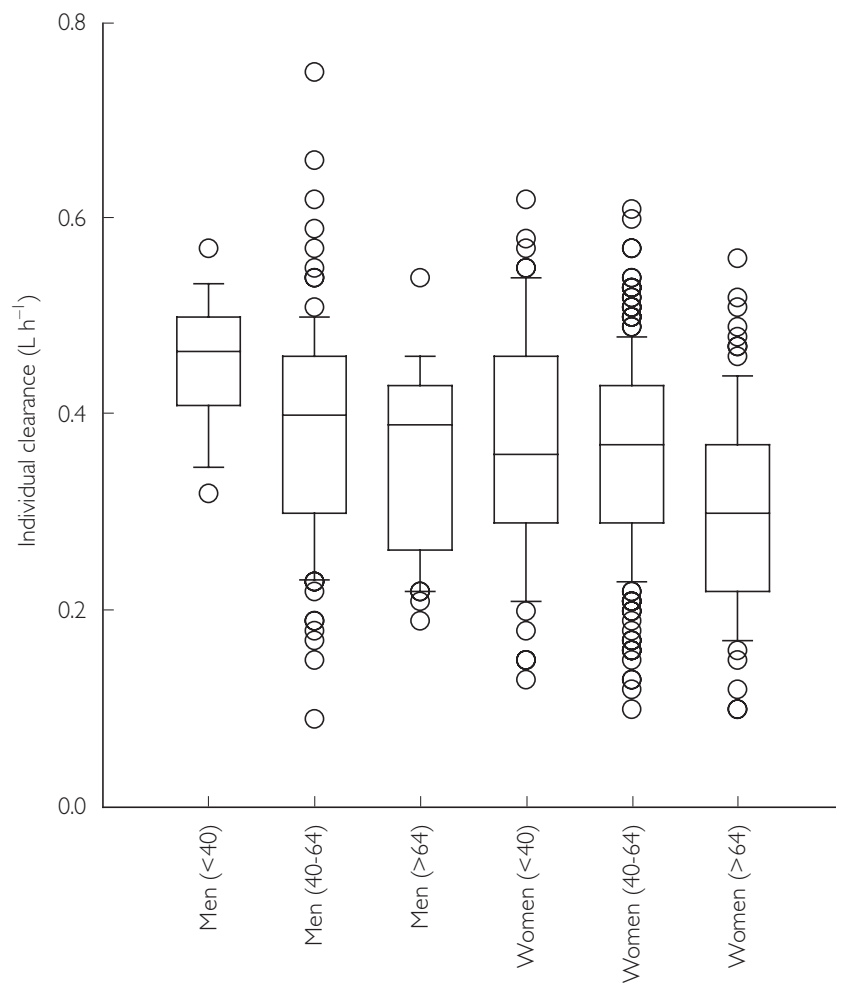

Figure 2 Age and gender dependence of individual post hoc clearance estimates. Box plots illustrate the median, interquartile range $(25-75 \%)$ and outliers $(<10 \%$ and $>90 \%)$. with $\mu[\mathrm{i}]$ regression parameters to be estimated and $K A^{\star}=K A-C L / V$.

With these definitions a population clearance for the mean female subject of $0.3471 \mathrm{~h}^{-1}$ was estimated and of $0.375 \mathrm{l} \mathrm{h}^{-1}$ for the male counterpart. The location parameter for volume was 17.51 and the absorption rate constant was $0.511 \mathrm{~h}^{-1}$ for male and $0.509 \mathrm{~h}^{-1}$ for female patients (Table 2).

Comedications occurring in $>5 \%$ of the patients were then tested as covariates for an effect on meloxicam clearance (Figure 3 ). The latter was not significantly affected $(P>0.05)$ by azathioprine $(n=33), \quad \beta-$ adrenoceptor blockers $(n=37)$, chloroquine $(n=45)$, d-

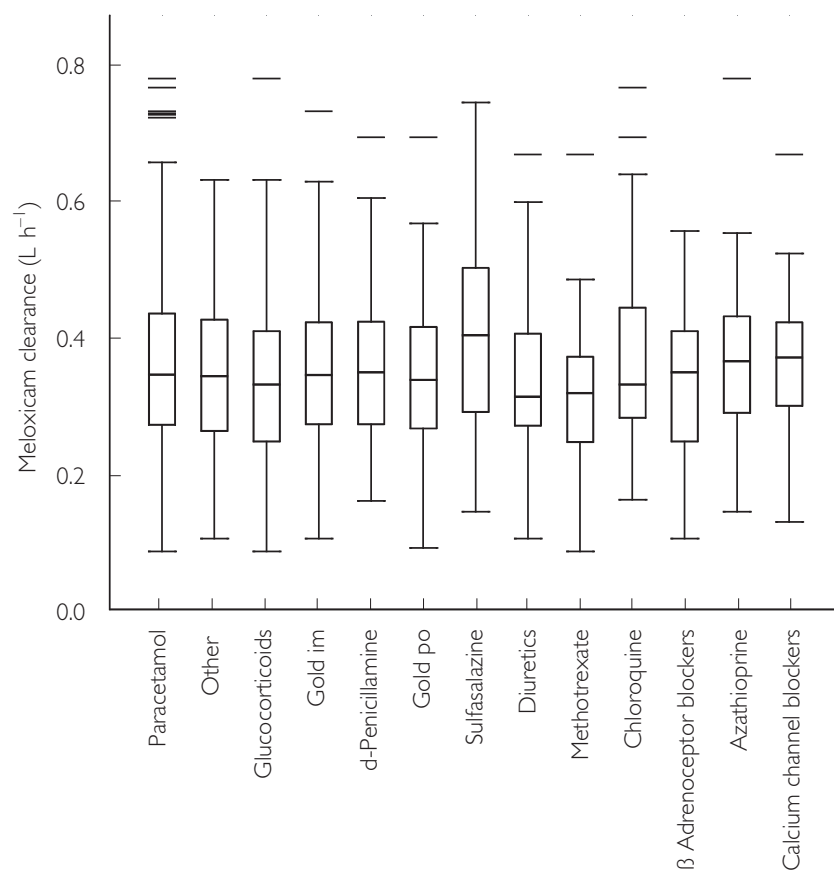

Figure 3 The effect of comedication on the clearance of meloxicam. Box plots of the groups with more than 30 patients show median and interquartile range (25-75\%) and outliers $(<10 \%$ and $>90 \%)$.

Table 2 Population pharmacokinetic estimates.

\begin{tabular}{|c|c|c|c|c|c|}
\hline & \multicolumn{2}{|c|}{ Clearance $\left(l h^{-1}\right)$} & Volume (l) & \multicolumn{2}{|c|}{$K A\left(h^{-1}\right)$} \\
\hline NONMEM estimate & 0.377 & 0.347 & 14.9 & 0.338 & 0.336 \\
\hline $95 \% \mathrm{CI}^{\star}$ & $0.304-0.449$ & $0.274-0.419$ & & $0.334-0.342$ & $0.332-0.340$ \\
\hline Individual estimates ${ }^{\dagger}(\min , \max )$ & $0.085-0.777$ & & $7.8-25.2$ & $0.06-1.37$ & \\
\hline WinBUGS estimate & 0.375 & 0.347 & 17.5 & 0.511 & 0.509 \\
\hline
\end{tabular}

${ }^{\star}$ Confidence interval: conventional confidence intervals define a range for the expectation of an estimate in frequently repeated experiments. †Individual estimates: range of individual parameter Bayesian posterior estimates from the final model. $\ddagger$ Credible interval: statement in the Bayesian framework concerning the probability of an unknown population parameter falling in the specified interval in this experiment. 
penicillamine $(n=84)$, diuretics $(n=51)$, methotrexate $(n=44)$ or paracetamol $(n=396)$. In addition, none of the drugs metabolized by CYP2C9 (sulphonamides, glibenclamide) or CYP3A4 (calcium channel blockers, erythromycin, benzodiazepines) had any statistically significant effect on clearance. A statistically significant effect $(P<0.005)$ on meloxicam clearance was observed following the coadministration of sulphasalazine (an increase of $19 \% ; n=54$ ) and glucocorticoids (a decrease of $12 \%$; $n=75)$. Gold comedication also affected meloxicam clearance (a decrease of $8 \% ; n=148$ ), but the large standard error of the parameter modelling the administration of gold led to $95 \%$ confidence intervals that included the null value (Table 3 ).

Similar trends were observed in the pharmacokinetic parameter estimates obtained using WinBUGS. The mean clearance was $0.421 \mathrm{~h}^{-1}$ in the sulphasalazine group, in $0.36 \mathrm{~h} \mathrm{~h}^{-1}$ for the glucocorticoid group, and $0.401 \mathrm{~h}^{-1}$ for the gold comedication group.

\section{Discussion}

Data from the present population pharmacokinetic analysis were similar to those obtained in phase I trials. Thus, the population mean estimate for clearance in males was $0.3771 \mathrm{~h}^{-1}$, which compares with a value of $0.4541 \mathrm{~h}^{-1}$ following multiple oral administration of meloxicam $(15 \mathrm{mg})$ to 24 healthy male volunteers [5]. Our NONMEM estimate for the volume of distribution (14.9 1) was also similar to that obtained in healthy male volunteers (13.8 1) [5]. This relatively small value results from the high protein-binding capacity of meloxicam (>99\%).

Analysis of covariates indicated that age appeared to be the most important factor influencing the pharmacokinetics of meloxicam. However, the effect of age was

Table 3 NONMEM regression parameters.

\begin{tabular}{llll}
\hline Parameter & Population estimate & s.e. & \\
\hline$\Theta_{1}(C L$, age $)$ & $0.1571 \mathrm{l} \mathrm{h}^{-1}$ & 0.0365 & \\
$\Theta_{2}(V$, weight $)$ & $0.2176 \mathrm{~kg}^{-1}$ & 0.0235 & \\
$\Theta_{3}(K A)$ & $0.3130 \mathrm{~h}^{-1}$ & 0.0578 & \\
$\Theta_{4}(C L$, weight $)$ & $0.00281 \mathrm{~kg}^{-1} \mathrm{~h}^{-1}$ & 0.0005 & \\
$\Theta_{5}(F 1$ bioav. $)$ & 0.9194 & 0.0185 & \\
$\Theta_{6}(C L$, age $)$ & -0.5169 & 0.0165 & \\
$\Theta_{7}(C L$, gender $)$ & $0.0342 \mathrm{lh}^{-1}$ & 0.0173 & \\
Sulphasalazine & 0.0652 & 0.0263 & delta $^{\dagger}: 13.8$ \\
Gold & -0.0280 & 0.0214 & delta $^{\dagger}: 4.0$ \\
Corticosteroids & -0.0426 & 0.0193 & delta $^{\dagger}: 9.9$ \\
\hline
\end{tabular}

$C L=\Theta_{1} .(\mathrm{AGE} / 50)^{\Theta 6}+\Theta_{4} \cdot W G T+\Theta_{7} \cdot(1-\mathrm{SEX}), V=\Theta_{2} . W G T$. $K A=C L / V+\Theta_{3} . \quad F 1=\Theta_{5} \quad . \quad(1-\mathrm{OCC})+$ OCC. $\star_{\text {s.e., }}$ estimated asymptotic standard error of the parameter. ${ }^{\dagger}$ Delta, difference in NONMEM objective function with and without inclusion of the covariate, $\mathrm{c}(P<0.05)=3.84$. relatively small and no dose adjustment was considered to be necessary. The increase in clearance associated with weight was accompanied by a similar increase in volume, resulting in an unchanged half-life. The observed differences in clearance between men and women can be attributed to differences in weight between the genders.

The analysis presented here supports previous studies, which reported a lack of interaction between meloxicam and methotrexate [21] or diuretics such as furosemide $[22,23]$. Although one study has demonstrated that coadministration of other NSAIDs produced significant decreases in the renal clearance of methotrexate given at maintenance doses [24], other studies have failed to find any significant pharmacokinetic interaction between methotrexate and NSAIDs in RA patients with adequate renal function [25-27]. A number of NSAIDs, including ibuprofen, naproxen, sulindac [28] and indomethacin [29], suppress prostaglandin-dependent renal blood flow and furosemide-induced diuresis. However, a pharmacodynamic interaction with furosemide may not be common to all NSAIDs. For example, although tenoxicam caused a significant decrease in prostaglandin $\mathrm{E}_{2}$ concentrations, the excretion profile of furosemide was not significantly changed by concomitant administration of tenoxicam [30]. The lack of a significant pharmacokinetic interaction between meloxicam and diuretics such as furosemide can possibly be explained by the assumption that classical NSAIDs and diuretics interact by COX-1dependent mechanisms, and because meloxicam preferentially inhibits COX-2, this interaction is minimized.

The mechanisms by which sulphasalazine and glucocorticoids affect meloxicam clearance remain to be elucidated. Meloxicam is mainly metabolized by CYP2C9 [3]. The increase in meloxicam clearance observed in the present study after coadministration of sulphasalazine suggests that induction of CYP2C9 has occurred.

Glucocorticoids induce the CYP3A subfamily [31], which includes CYP3A4, a minor contributor to the metabolism of meloxicam [3]. Induction of CYP3A4 by glucocorticoids might be expected to result in a small increase in meloxicam clearance, not the decrease observed in this study. It is possible that glucocorticoids may act as competitive inhibitors of drug metabolizing enzymes. The clinical significance of the observed changes in the clearance of meloxicam is not certain.

Only limited information is available on the comparative performance of NONMEM and Markov chain Monte Carlo methods [18, 32]. Such a comparison is very demanding of processor speed and random access memory. The WinBUGS package together with the PKBUGS add-on makes the ability to perform full Bayesian population pharmacokinetic analyses more generally available. The location parameters obtained for our data set with both programs are in reasonable agreement and 
the Bayesian 95\% credible intervals (Table 2) appear to be similar to the conventional confidence intervals reflecting the variability in the data, although both statistical ranges are conceptually not identical. WinBUGS in its present form complements existing established procedures and we are confident that the built-in extendibility of the concept will soon lead to a less constrained package that is as universally applicable as NONMEM.

In conclusion, the population pharmacokinetic analysis of data from patients with RA participating in meloxicam trials gave similar results to those obtained from phase I trials. A typical female patient with a body weight of $58.5 \mathrm{~kg}$ and an age of 64.5 years is predicted to have a clearance of $0.301 \mathrm{~h}^{-1}$. In contrast, in a typical male patient with $78.5 \mathrm{~kg}$, aged 44.5 years a clearance of $0.421 \mathrm{~h}^{-1}$ is expected. Sulphasalazine and glucocorticoid coadministration resulted in changes in meloxicam clearance of $+19 \%$ and $-12 \%$, respectively, the mechanisms of which are unknown. The pharmacokinetics of meloxicam appeared to be unaffected by many other common comedications such as methotrexate, azathioprine, $\beta$ adrenoceptor blockers, diuretics, chloroquine, Dpenicillamine, sulphonamides, glibenclamide, calcium channel blockers, erythromycin and benzodiazepines. However, clinically important interactions might occur rarely and may not be detected in phase I trials due to the relatively small number of observations in these studies. Therefore, patients who are taking meloxicam with any comedication should be carefully monitored.

This study was supported by Boehringer Ingelheim (Germany).

\section{References}

1 Degner F, Türck D, Pairet M. Pharmacological, pharmacokinetic and clinical profile of meloxicam. Drugs Today 1997; 33: 739-758.

2 Türck D, Busch U, Heinzel G, Narjes H, Nehmiz G. Effect of food on the pharmacokinetics of meloxicam after oral administration. Clin Drug Invest 1995; 9: 270-276.

3 Chesné C, Guyomard C, Guillouzo A, Schmid J, Ludwig E, Sauter T. Metabolism of meloxicam in human liver involves cytochromes P4502C9 and 3A4. Xenobiotica 1998; 28: 1-13.

4 Schmid J, Busch U, Heinzel G, Bozler G, Kaschke S, Kummer M. Pharmacokinetics and metabolic pattern after intravenous infusion and oral administration to healthy subjects. Drug Metab Dis 1995; 23: 1206-1213.

5 Türck D, Busch U, Heinzel G, Narjes H. Clinical pharmacokinetics of meloxicam. Arzneimittelforschung 1997; 47: 253-258.

6 Hobbs DC. Piroxicam pharmacokinetics: recent clinical results relating kinetics and plasma levels to age, sex, and adverse events. Am J Med 1986; 81 (Suppl 5B).

7 Verbeeck RK, Richardson CJ, Blocka KLN. Clinical pharmacokinetics of piroxicam. J Rheumatol 1986; 13: 789796.
8 Crevoisier C, Heizmann P, Forgo I, Dubach UC. Plasma tenoxicam concentrations after single and multiple oral doses. Eur J Drug Metab Pharmacokinet 1989; 14: 23-27.

9 Hosie J, Distel M, Bluhmki E. Efficacy and tolerability of meloxicam versus piroxicam in patients with osteoarthritis of the hip or knee: a six-month double-blind study. Clin Drug Invest 1997; 13: 175-184.

10 Goei Thè HS, Lund B, Distel MR, Bluhmki E. A doubleblind randomised trial to compare meloxicam $15 \mathrm{mg}$ with diclofenac $100 \mathrm{mg}$ in the treatment of osteoarthritis of the knee. Osteoarthr Cartil 1997; 5: 283-288.

11 Lemmel EM, Bolten W, Burgos-Vargas R, Platt P, Nissilä M, Sahlberg D. Efficacy and safety of meloxicam in patients with rheumatoid arthritis. J Rheumatol 1997; 24: 282-290.

12 Brooks P. Rheumatology. BMJ 1988; 316: 1810-1812.

13 Reginster JY, Distel M, Bluhmki E. A double-blind, three-week study to compare the efficacy and safety of meloxicam $7.5 \mathrm{mg}$ and meloxicam $15 \mathrm{mg}$ in patients with rheumatoid arthritis. Br J Rheumatol 1996; 35 (Suppl 1): 1721.

14 Degner FL, Huskisson EC, Narjes H, Bluhmki E. Randomized double-blind clinical trial over 3 weeks comparing meloxicam $15 \mathrm{mg}$ with piroxicam $20 \mathrm{mg}$ in rheumatoid arthritis. $Z$ Rheumatol 1996; 55 (Suppl 1): 140.

15 Ropes MW, Bennet GA, Cobb S, Joax R, Jessar RA. 1958 revision of diagnostic criteria for rheumatoid arthritis. Bull Rheum Dis 1958; 9: 175-176.

16 Arnett FC, Edworth SM, Block DA. The American Rheumatism Association 1987, revised criteria for the classification of rheumatoid arthritis. Arthritis Rheum 1993; 31: 315-324.

17 Beal SL, Sheiner LB, eds. NONMEM Users Guides. San Francisco: NONMEM Project Group University of California, 1998.

18 Lunn DJ, Thomas A, Best N, Spiegelhalter D. WinBUGSa Bayesian modelling framework: concepts, structure and extensibility. Statistics Computing 2000; 10: 325-337.

19 Beal SL, Sheiner LB, eds. NONMEM Users Guide V. San Francisco: NONMEM Project Group University of California, 1998: 48.

20 Mandema JW, Verotta D, Sheiner LB. Building population pharmacokinetic pharmacodynamic models. Models for covariate effects. J Pharmacokin Biopharm 1992; 20: 511-528.

21 Hübner G, Sander O, Degner FL, Türck D, Rau R. Lack of pharmacokinetic interaction of meloxicam with methotrexate in patients with rheumatoid arthritis. $J$ Rheumatol 1997; 24: 845-851.

22 Müller FOR, Schall R, deVaal AC, Groenewoud G, Hundt HKL, Middle MV. Influence of meloxicam on furosemide pharmacokinetics and pharmacodynamics in healthy volunteers. Eur J Clin Pharmacol 1995; 48: 247-251.

23 Müller FO, Middle MV, Schall R, Terblanché J, Hundt HKL, Groenewoud G. An evaluation of the interaction of meloxicam with frusemide in patients with compensated chronic cardiac failure. Br J Clin Pharmacol 1997; 44: 393-398.

24 Kremer JM, Hamilton RA. The effects of nonsteroidal antiinflammatory drugs on methotrexate (MTX) pharmacokinetics: impairment of renal clearance of MTX at weekly maintenance doses but not at $7.5 \mathrm{mg}$. J Rheumatol 1995; 22: 2072-2077.

25 Ahern M, Booth J, Loxton A, McCarthy P, Meffin P, Kevat 
S. Methotrexate kinetics in rheumatoid arthritis. Is there an interaction with nonsteroidal antiinflammatory drugs? $J$

Rheumatol 1988; 15: 1356-1360.

26 Skeith KJ, Russell AS, Jamali F, Coates J, Friedman H. Lack of significant interaction between low dose methotrexate and ibuprofen or flurbiprofen in patients with arthritis. $J$

Rheumatol 1990; 17: 1008-1010.

27 Stewart CF, Fleming RA, Arkin CR, Evans WE.

Coadministration of naproxen and low-dose methotrexate in patients with rheumatoid arthritis. Clin Pharmacol Ther 1990; 47: 540-546.

28 Brater DC, Anderson S, Baird B, Campbell WB. Effects of ibuprofen, naproxen, and sulindac on prostaglandins in men. Kidney Int 1985; 27: 66-73.
29 Daskalopoulos G, Kronborg I, Katkov W, Gonzalez M, Laffi G, Zipser RD. Sulindac and indomethacin suppress the diuretic action of furosemide in patients with cirrhosis and ascites: evidence that sulindac affects renal prostaglandins. Am J Kidney Dis 1985; 6: 217-221.

30 Hartmann D, Kleinbloesem CH, Lücker PW, Vetter G. Study on the possible interaction between tenoxicam and furosemide. Arzneimittelforschung 1987; 37: 1072-1076.

31 Beyeler C, Frey BM, Bird HA. Urinary 6 $\beta$-hydroxycortisol excretion in rheumatoid arthritis. Br J Rheumatol 1997; 36: 54-58.

32 Best NG, Tan KKC, Gilksa WR, Spiegelhalter DJ. Estimation of population pharmacokinetics using the Gibbs sampler. J Pharmacokinet Biopharm 1995; 23: 407-435. 